\title{
Effective Morphological Transformation and Sub-pixel Classification of Clustered Images
}

\author{
Mr. B. Naga Rajesh \\ Assistant professor-ECE, Dr.K.V.Subba Reddy College of Engineering for Women, \\ nagarajesh87@gmail.com
}

\begin{tabular}{|l|l|}
\hline Article History & \multicolumn{1}{c|}{ Abstract } \\
$\begin{array}{l}\text { Article Submission } \\
22 \text { November } 2018 \\
\text { Revised Submission } \\
26 \text { January } 2019 \\
\text { Article Accepted } \\
7 \text { March } 2019 \\
\text { Article Published } \\
3 I^{\text {st }} \text { March } 2019\end{array}$ & $\begin{array}{l}\text { The main aim of this research work is to perform the morphological operations with } \\
\text { reduced time complexity and area complexity. Morphological operation is the key } \\
\text { element in any image processing. Finding the maximum and minimum using a } \\
\text { window of defined size will imply to the morphological dilation and erosion } \\
\text { respectively. So the proposed algorithm should be fast in the comparison and sorting, } \\
\text { this way the time complexity could be reduced. It's believed that the anchor concept } \\
\text { will fetch this cause. The idea behind this is it fixes a pixel and setting it as the center } \\
\text { pixel all the surrounding pixels will be processed. Moreover this is now been } \\
\text { implemented for rectangular structuring element. This paper attempts the same for } \\
\text { flat and 3D structuring elements. Hyper-spectral Imaging is a developing zone of } \\
\text { remote detecting applications. Hyper-spectral pictures incorporate more extravagant } \\
\text { and better otherworldly data than the multi-spectral pictures got previously. Hyper- } \\
\text { otherworldly pictures are described by an exchange off between the unearthly and } \\
\text { spatial resolution. The principle issue of the hyper-ghostly information is the } \\
\text { generally low spatial goal. For arrangement, the serious issue brought about by low } \\
\text { spatial goal is the blended pixels. Blended pixels alluded to the pixels which are } \\
\text { involved by more than one land spread class. In the proposed procedure another } \\
\text { strategy is utilized to address the issue of blended pixels and to get a better spatial } \\
\text { goal of the land spread characterization maps. The strategy misuses the upsides of } \\
\text { both picture bunching methods and phantom dimming calculations, so as to decide } \\
\text { the fragmentary plenitudes of the classes at a sub-pixel scale. Spatial regularization } \\
\text { by Flank planning method is at last performed to spatially find the got classes at sub- } \\
\text { pixel level. } \\
\text { Keywords: Mathematical morphology, erosion, Spatial resolution improvement, } \\
\text { dilation, Hyper spectral data, Image clustering, Spatial regularization }\end{array}$ \\
\hline
\end{tabular}

\section{Introduction}

Land spread arrangement of remote detecting information is a significant utilization of picture examination, and it has numerous viable applications. The persistently developing accessibility of hyper spectral symbolism has opened additional opportunities in the field of picture investigation and arrangement. Hyper spectral sensors are portrayed by a high spectral goal and a spatial goal which can differ from not many to several meters. One of the significant issues of hyper spectral pictures is the moderately low spatial goal, particularly in the event of high height sensors which spread wide regions. These sensor constraints can influence the exhibitions of calculations used to process hyper spectral information. In grouping, the moderately low spatial goal can prompt the difficult issue of blended pixels, i.e., pixels containing more than one land spread sort. Additionally if there should arise an occurrence of high spatial goal, a hyper spectral picture is regularly a blend of unadulterated and blended pixels [1].

The issue of blended pixels has been considered in a few works which utilizes a high spatial goal picture together with the low goal picture, so as to get an intertwined picture with high spectral and spatial goal, super- 
goal approaches, for example, fluffy classifiers autonomous from any high spatial goal information were additionally proposed. In any case, fluffy arrangement is a tedious strategy if there should be an occurrence of high spectral goal information. Also, these delicate grouping strategies don't dole out pixel to a solitary class. They produce set of pictures by making a level of enrolment. This level of participation doesn't mirror the fragmentary bounty of the class inside a blended pixel. The likelihood of a pixel have a place with one class doesn't really compare to the fragmentary piece of the pixel secured by that class [2].

Numerical Morphology is an instrument for removing picture parts that are helpful for portrayal and depiction. The method was initially evolved by Matheron and Serra at the Ecole des Mines in Paris. Scientific morphology utilizes ideas from set hypothesis, geometry and geography. This type of picture examination gives a quantitative portrayal of geometrical structures. The administrators are especially helpful for the examination of paired pictures and basic uses incorporate edge discovery, commotion expulsion, picture upgrade and picture division. It is additionally valuable for some pre-and post-preparing procedures, particularly in edge diminishing and pruning. As a rule most morphological activities depend on straightforward growing and contracting tasks. numerous calculations have been proposed to accelerate the calculation of morphological administrators. These calculations can be extensively isolated into two families: (1) calculations that misleadingly diminish the size of the organizing set or capacity used to contrast a sign and, through an examination and deterioration of the organizing set or capacity into littler pieces, and (2) calculations that investigate the sign to decrease the quantity of repetitive correlations natural to definitions like the morphological disintegration. The essential use of morphology happens in double pictures, however it is likewise utilized on dark level pictures [3].

Scientific morphology is primarily with the end goal of article or imperfection distinguishing proof required in numerous applications. Robert M. Haralick, Stanley R. Sternberg and Xinhua Zhuang in [1] give a detailed blueprint on twofold and dim scale morphological activities. Numerical morphology is mostly founded on set hypothesis. Marc Van Droogebbroeck and Hugues Talbot in [4] show scientific morphology as the field of picture preparing which centers basically around the shape investigation of article and capacities. Dinu Coltus and Ioannis Pitas in [3] clarify about the quick running max-min channels for self-assertive size windows. The size of the window is being expanded and the info arrangement is likewise being extended. The calculation multifaceted nature of the calculation is seen as near $\log 2 n$, where ' $n$ ' is the size of the window. Ioannis Pitas in [4] manages channel preparing. In channel handling the request measurements are utilized broadly, in light of their calculation speed. Scott C. Douglas in [5] presents a calculation for the count of running most extreme and least estimation of a 1D succession over a sliding information window.

Mike Brookes in [6] has introduced a calculation for the execution of a running max/min channel of subjective request $\mathrm{K}$, the normal calculation time per test is asymptotically autonomous of $\mathrm{K}$. Running max and min channels are generally utilized in the handling of discourse and picture signals in light of their vigorous conduct within the sight of commotion and sign non-stationarities. Thomas S. Huang, George J. Yang and Gregory Y. Tang in [7] present a quick calculation enemy 2D middle separating. It depends on arranging and refreshing the dim level histogram of the image components in the calculation is of the request for O(n). David Z. Gevorkian, Jaakko T. Astola and Samvel M. Atourian in [8] state about the best bound on the quantity of examinations expected to register the running max/min over a ' $\mathrm{p}$ '- component sliding window is roughly three. Joseph (Yossi) Gil and Ron Kimmel in [9] a powerful calculation for numerical morphology are being clarified. Calculation here introduced is a combination of the past Van Herk and Gil and Werman calculation. Joseph Gil and Michael Werman in [10] give a quick calculation to discover min, middle, max or some other measurement channel changes. The calculation sets aside steady effort for min and max channels and polylog time for middle channel.

\section{Existing techniques}

Various methods were created abusing the factual and geometrical properties of the information. Yet, these strategies can in part defeat the disadvantage of the full pixel strategy. So in the proposed strategy a blend of hard order and spectral immixing is utilized to get a fresh sub-pixel characterization map. Hyper spectral information contains numerous pixels which have a place with one class and numerous others with blended 
class. In this way the mix of these procedures can be viewed as an intriguing and correlative methodology for sub-pixel order of hyper spectral information. Followed by spectral immixing, sub-pixel planning is gotten by Flank planning strategy [5][7].

The proposed procedure incorporates three stages, for example, extraction of spectral portrayal of the classes by methods for ISODATA hard arrangement strategy. Followed by this Fully Constrained Least Square Spectral Immixing is done to locate the fragmentary wealth of the classes present in every pixel. The unmixed information is given as contribution to the Flank planning procedure to spatially find the classes inside the subpixel. Numerical morphology inspects the geometrical structure of a picture by testing it with little examples, called 'organizing components', of fluctuating size and shape, only the manner in which a visually impaired man investigates the world with his fingers or a stick. This method brings about nonlinear picture administrators which are appropriate to investigating geometrical and topological structures. A progression of such administrators is applied to a picture so as to make certain highlights clear, recognizing important data from unessential twists, by diminishing it to such a cartoon. For instance, in optical character acknowledgment one may change the advanced picture of an image by decreasing each associated segment to a one-pixel-thick skeleton holding the image's shape. Such a skeleton does the trick for acknowledgment and can be taken care of substantially more monetarily than the full image [9].

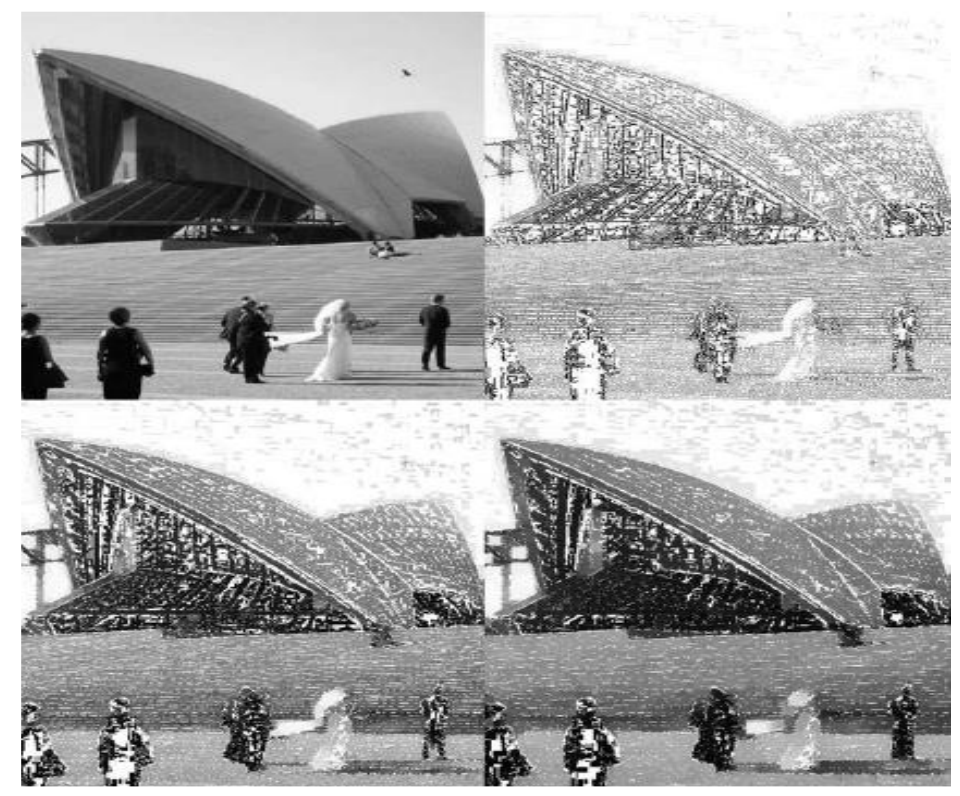

Fig 1: Basic input image with sub-pixel classification

The two most fundamental activities in numerical morphology are disintegration and enlargement. Both of these administrators accept two bits of information as information: a picture to be dissolved or widened, and an organizing component (otherwise called a bit). The two bits of info information are each rewarded as speaking to sets of directions in a manner that is marginally extraordinary for paired and dim scale pictures. For a double picture, white pixels are regularly taken to speak to closer view districts, while dark pixels signify foundation. At that point set of directions comparing to that picture is basically the arrangement of two-dimensional Euclidean directions of all the frontal area pixels in the picture, with a cause ordinarily taken in one of the corners so all directions have positive components. These changes include the association between a picture A (the object of intrigue) and an organizing set $\mathrm{B}$, called the organizing component. Ordinarily the organizing component $\mathrm{B}$ is a round circle in the plane, however it very well may be any shape. The picture and organizing component sets need not be confined to sets in the 2D plane, yet could be characterized in 1, 2, 3 (or higher) measurements [10]. 


\section{Proposed Methodology}

The hyper spectral data is given as input to the hard classification method. ISODATA clustering technique is the hard classification technique used here. The result of this step is the cluster representation of the classes. It gives the spectral representation of the pure pixels present in the image. The spectral representations of the classes are determined by taking the centroid of the clusters along each band. The results of this step along with the hyper spectral data are given as input to the spectral immixing technique. Here fully constrained linear spectral immixing technique is used. Several algorithms are developed to handle linear immixing, but failed satisfy both full additively and nonnegative constraints. Fully constrained linear immixing technique satisfies both fully additively and no negativity constraints. The unmixed data is given as input to flank mapping method. The work flow is shown in figure 2.

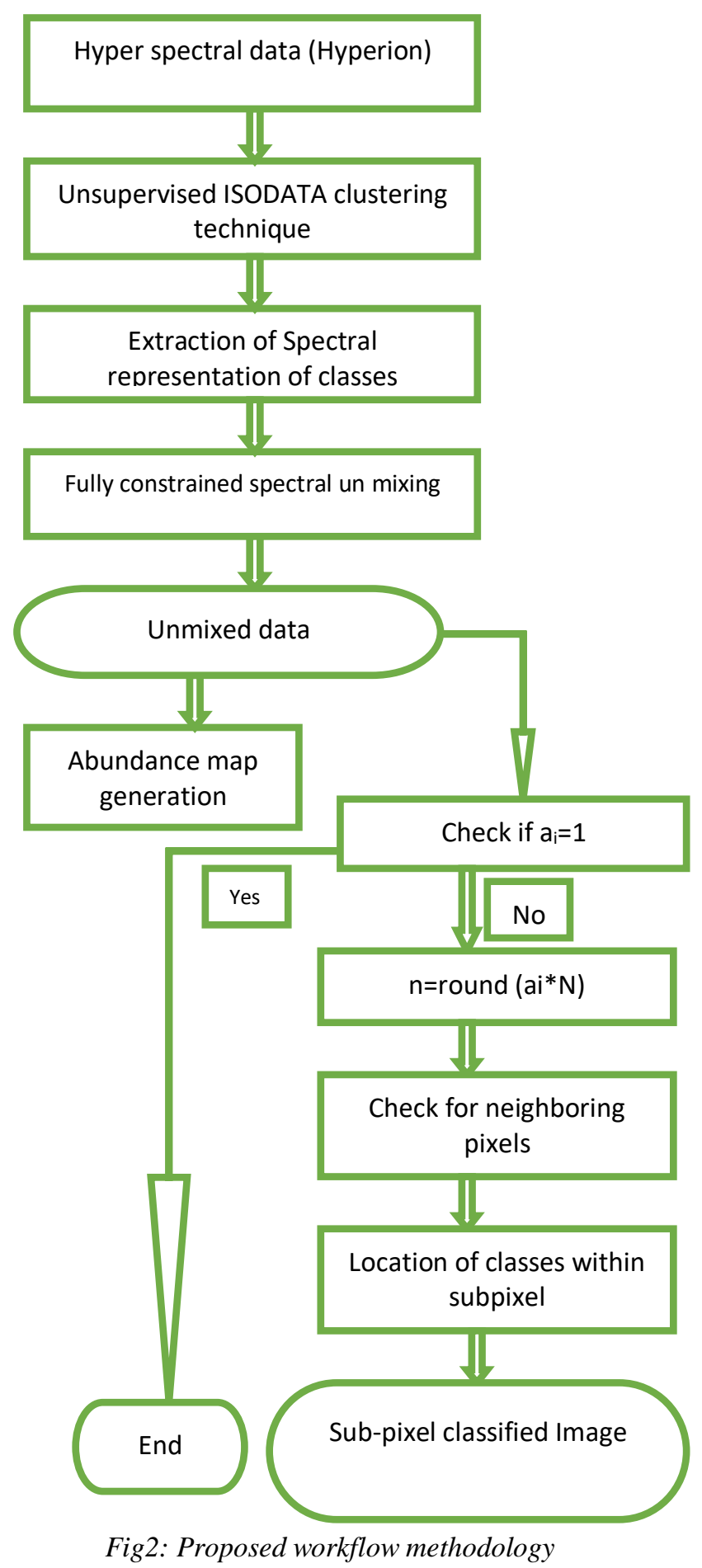


ISODATA clustering is an unsupervised hard classification technique. It is an iterative algorithm. First assigns arbitrary initial cluster vector. Next it classifies each pixel, by measuring the distance between the mean cluster vectors and each cluster. The steps are repeated until there are no changes between the successive iterations. The centroids of the resultant clusters are retained as the spectral representation of each class.

Spectral un-mixing technique is used to determine the weight of presence of each class within a pixel. Fully Constrained linear mixing technique is used here. This technique satisfies two main constraints such as sum to one and non negativity constraint. The sum of fractional abundance of all classes within a pixel should be equal to one and the fractional abundance of the class should not be less than zero $(0)$. The weight of each class within a pixel is proportional to the pixel area covered by that class. If there is ' $\mathrm{K}$ ' number of spectral bands, the spectrum of the classes is represented by K-dimensional vectors. The general equation for linear spectral unmixing is given by,

$\mathrm{Z}=\mathrm{Kx} 1$ pixel vector

$$
\mathrm{Z}=\sum \mathrm{i}=1 \mathrm{~L} \text { aisi }
$$

$\mathrm{i}=1,2, \ldots \mathrm{L}$

$\mathrm{L}=$ No. of classes

ai=Abundance of ith class

$\mathrm{Si}=[\mathrm{s} 1, \mathrm{~s} 2, \mathrm{~s} 3, \ldots \mathrm{sL}]$

$\mathrm{S}$ is the spectral representation of the classes which is of $\mathrm{KxL}$ dimension.

Then the classes are spatially located in the sub-pixels by the newly proposed Flank mapping method. If abundance value of a particular pixel is equal to one then that pixel is wholly assigned to a single class. Else by using the formula in equation (2) the no. of sub pixels to be assigned to a class is found by giving the abundance value determined from the un-mixing step. Then the neighbouring pixels are checked to spatially locate the classes within the sub-pixels. Since it checks adjacent pixels for mapping classes it is known as Flank mapping technique. The resultant is the sub-pixel classified map. Configuration 1 and 3 are unable to determine new anchor. Configuration 1 results in either one of type 3 or 4 . Configuration 3 results any one of types $4,5 \& 6$. The algorithm determines the opening anchor by looking for the minimum of $f[x 1] \ldots . . f[x N]$. Configuration 5 is the unfortunate condition where the opening anchor could not be found in one hop. Once the anchor is found then opening is calculated by the following steps.

Analyzing the image in the 2D format is much difficult and a time consuming process so the initial preprocessing step is to convert the problem image to $1 \mathrm{D}$ format. Morphological operation in $1 \mathrm{D}$ format is much simpler than the multidimensional format. Once the image is been converted to the $1 \mathrm{D}$ format all the storage locations has to be initialized. The processing the image starts from the left and moves towards the right. The initializations needed for the start of this algorithm are as follows; ' $f$ ' input image; ' $g$ ' output buffer; $g[0]=f$ $[0]$.

\section{Simulation Results}

The hyper spectral data collected by Hyperion sensor of Earth observation-1 (Eo-1) satellite is used for analysis. The study area covered by the data extends from $13.00663468 \mathrm{~N}$ latitude, $79.97978483 \mathrm{E}$ longitudes in the upper left to $12.88917381 \mathrm{~N}$ latitude, $80.02480785 \mathrm{E}$ longitudes in the lower right. Hyper spectral data of the study area is shown in Figure 3. The study area consists of water bodies, urban, roads, vegetation, irrigation lands. The spectral representation of the classes obtained from ISODATA clustering technique is shown in Figure 3. The abundance map generated from linear spectral un-mixing step is shown in Figure 5a, 5b, 5c, 5d, 5e. The quantity of presence of land cover class in the pixel is shown by the degree of variation of brightness. The brighter portions in the map show the $100 \%$ presence of that land cover class in that pixel and the darker portions denote the absence of that class. Similarly for each class an abundance map is generated. Using the unmixed data the classes are spatially located in the sub-pixels by flank mapping technique. 


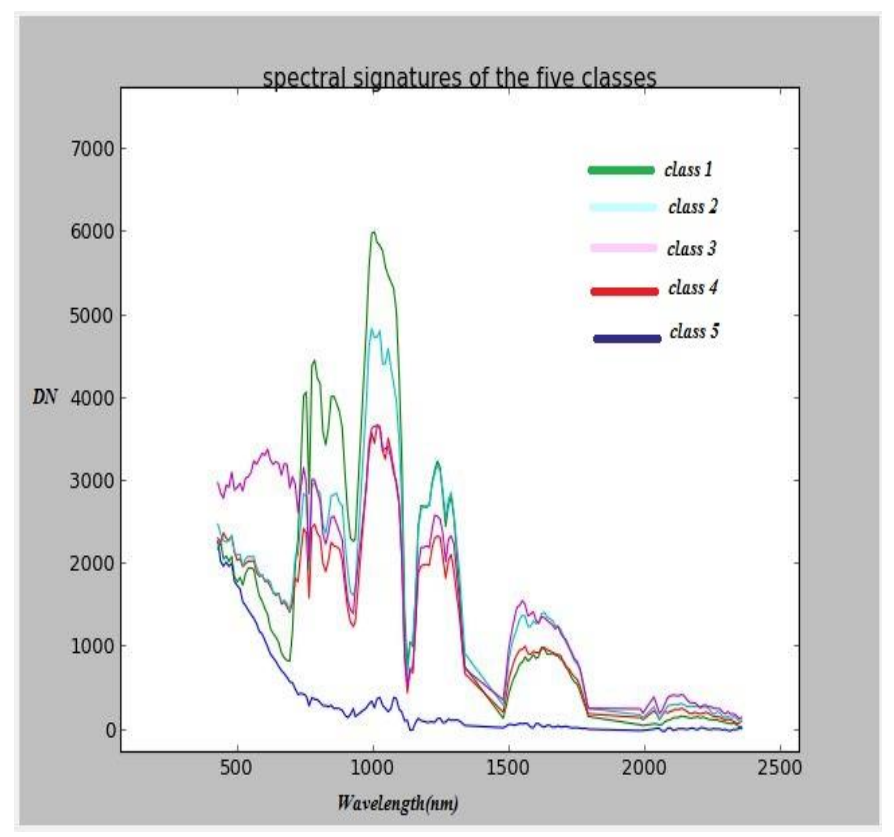

Fig 3: Spectral representation of the five classes.

This project is been carried out in two steps. The first one is finding the locations of the anchor, and then finding the opening using the anchor location found in the previous step. The input image given is any image on which the morphological function has to be performed. If the original image is been used for the computation as such then the computation complexity becomes a serious problem so the actual problem image is been resized. Once the figure is been resized then all the steps of the algorithm has to be performed one by one. The first one is converting the $2 \mathrm{D}$ image into the $1 \mathrm{D}$ image. The conversion of the problem image to $1 \mathrm{D}$ is the pre processing step after that the algorithm steps get started. The first thing will be the global copy, which is helpful in reducing the computation time of the algorithm.

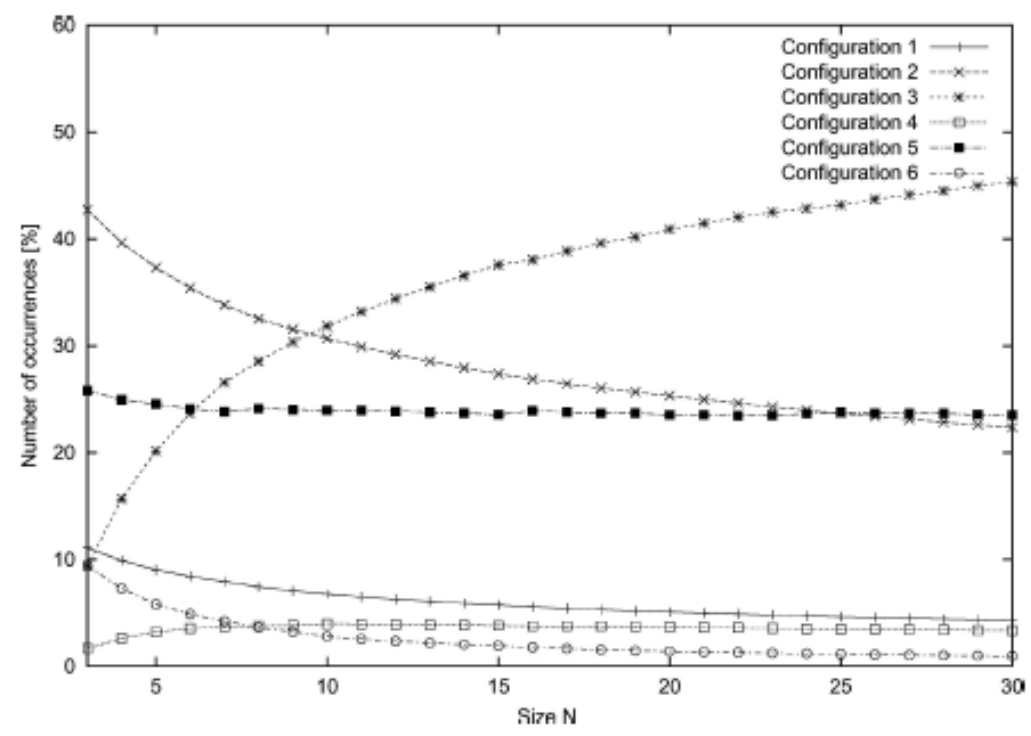

Fig4: Number of occurrences for each configuration for a natural image

After this the comparison of the input will be done with the output buffer. If there is a change in the value of the comparison then an update will be done or else the same values will be retained. Thus these minimum value locations found by this algorithm gives the anchor points. 


\section{Conclusion}

The sub-pixel classified is checked by ground truth verification. The verification results show that the classification is highly accurate. The hard classification technique followed by spectral unmixing and flank mapping is computationally efficient and gives very high accurate results when compared with conventional technique. Morphological operation is one of the effective tools in the image processing scheme. Here in this algorithm that process has been carried out even effectively. Some of the results of this algorithm are being listed down. The pre-processing step of converting the problem image to 1D format also did contribute for the speedy computation of the process. Thus by this algorithm the total run time is around $0.8 \mathrm{sec}$. This is observed to be $30 \%$ over the other algorithms (van Herk's algorithm and Gil-Kimmel's algorithm). Due to the 'Global copy' step in the algorithm the storage space is much reduced. Ultimately evaluating the morphological operation using the anchor concept is much effective.

\section{References}

[1] Brookes. M (2000), "Algorithms for max and min filters with improved worst-case performance," IEEE Transactions on Circuits and Systems II: Analog and Digital Signal Processing,Vol. 47, No. 9, pp. 930-935.

[2] Coltuc. D and Pitas. I (1997), "On fast running max-min filtering," IEEE Transactions on Circuits and Systems II: Analog and Digital Signal Processing, Vol. 44, No. 8, pp. 660-663.

[3] Douglas. S (1996), "Running max/min calculation using a pruned ordered list," IEEE Transactions on Signal Processing, Vol. 44, No. 11, pp. 2872-2877.

[4] Gevorkian. D, Astola. J, and Atourian. S (1997), "Improving Gil-Werman algorithm for running min and max filters," IEEE Transactions on Pattern Analysis and Machine Intelligence, Vol. 19, No. 5, pp. 526-529.

[5] Gil. J and Kimmel. R (2002), "Efficient dilation, erosion, opening, and closing algorithms," IEEE Transactions on Pattern Analysis and Machine Intelligence, Vol. 24, No. 12, pp. 1606-1617.

[6] San B.T and M.L.Suzen (2010), "Evaluation of different atmospheric correction algorithms for EO-1 Hyperion image", International archives of the photogrammetric, Remote sensing and spatial Information Science, Vol.XXXVIII, Part 8, Kyoto, Japan.

[7] Villa. A et al., (2011), "Unsupervised classification and spectral unmixing for subpixel labeling", Geoscience and Remote sensing symposium (IGRASS), IEEE international, pp: 71 - 74.

[8] Daniel .C. Heinz and C.I.Chang (2001), "Fully constrained least square linear mixture analysis method for material quantification in hyperspectral imagery", IEEE transactions on Geoscience and Remote sensing, Vol.39, pp.529-545.

[9] C. Yuan, M. Zhou, L. Sun, S. Qiu and Q. Li, "A Multi-Dimensional Hyperspectral Image Mosaic Method and its Acquisition System," 2018 11th International Congress on Image and Signal Processing, BioMedical Engineering and Informatics (CISP-BMEI), Beijing, China, 2018, pp. 1-6, doi: 10.1109/CISP-BMEI.2018.8633038.

[10] N. Heide, C. Frese, T. Emter and J. Petereit, "Real- Time Hyperspectral Stereo Processing for the Generation of 3D Depth Information," 2018 25th IEEE International Conference on Image Processing (ICIP), Athens, 2018, pp. 3299-3303, doi: 10.1109/ICIP.2018.8451194. 